\title{
Publisher Correction: Visual perception: Sensory category learning
}

Stefan Pollmann

Correction to: Nature Human Behaviour https://doi.org/10.1038/s41562-018-0377-5, published online 2 July 2018.

In the version of this News \& Views originally published, the following sentence was mistakenly omitted from the Fig. 1 caption: 'Gabor patches generated from https://www.cogsci.nl/gabor-generator under a Creative Commons licence CC BY 4.0.' This has now been corrected. 